\title{
Determination of the spherical part of the couple-stress in a polar fibre-reinforced elastic plate subjected to pure bending
}

Received: 14 January 2021 / Revised: 21 April 2021 / Accepted: 29 May 2021 / Published online: 24 July 2021 (C) The Author(s) 2021

\begin{abstract}
This communication provides initial information and understanding of the manner in which a newly developed theoretical mechanism (Soldatos in Int J Solids Struct 202:217-225, 2020) is applied in specific boundary value problems met in polar linear elasticity of fibrous composites and thus enables the determination of the spherical part of the couple-stress tensor. In this context, it tests the applicability of the implied mechanism/method in the case that a rectangular plate reinforced by a single family of unidirectional fibres is subjected to pure bending. The problem solution is obtained for either non-polar or polar material behaviour, where fibres are considered perfectly flexible or resistant in bending, respectively, and provides clear evidence of the correctness of the principal argument that underpins the proposed method. Namely, that the general rotation field of the plate deformation differs from the fibre rotation field. That newly discovered method enables an extra energy term that emerges in the strain energy function of the fibrous composite plate to relate with the spherical part of the couple-stress tensor outside conventional equilibrium conventions. It thus leads to the determination of the spherical part of the couple-stress and its distribution throughout the plate body in a complete and comprehensive manner.
\end{abstract}

\section{Introduction}

It is well known that the spherical part of the couple-stress tensor does not affect the stress equilibrium and is, therefore, left indeterminate in conventional polar elasticity (e.g. [1-11] and references therein). On the other hand, though, the polar elasticity theory of fibre-reinforced materials [7, 9] reveals that the strain energy density/function of polar fibrous composites contains an extra energy term that also leaves unaffected their stress equilibrium. These observations led the present author [12] to search for a mechanism that could connect the implied extra energy term with the work done by the spherical part of the couple-stress and thus would enable determination of the latter outside or regardless of standard equilibrium conventions.

The search for such a mechanism made it initially understood [12] that the implied indeterminacy arises by the direct manner in which the Cosserat framework [13], which underpins the conventional theory of polar elasticity, relates the spherical part of the couple-stress tensor to the rotation field of the deformation. This rotation field, $\omega$, is the antisymmetric part of the displacement gradient and is generally present in a deformation regardless of whether the solid of interest exhibits polar or non-polar material behaviour. In the latter case though, which embraces non-polar linear elasticity, the interaction of this antisymmetric rotation field with the encountered symmetric stress field does not produce work and, hence, does not influence the strain energy function. In other words, the Cosserat theoretical framework essentially considers that the general rotation field of deformation, $\omega$, relates to the emerged couple-stress field in a manner that makes it reciprocal to 
its associated antisymmetric stress counterpart. Hence, necessarily, the latter contributes to the strain energy function of the polar material of interest only by its direct interaction with the antisymmetric field $\omega$.

However, in the case of polar material behaviour of fibrous composites [7, 9], where fibres with bending stiffness behave like embedded Euler-Bernoulli beams, no apparent reason requires from the resulting fibre rotation field, $\varphi$ say, to coincide with for the corresponding general rotation field of the deformation. This fact led the present author [12] to generalise the Cosserat couple-stress theory in a manner that enables distinction of the two different rotation fields $\omega$ and $\varphi$. The implied theoretical generalisation [12] considers that, at least in the case of polar fibrous composites [7, 9], it is the fibre rotation field, rather than its general deformation counterpart, that is reciprocal to the antisymmetric part of the stress. In this manner, (i) it creates room for a direct connection to be made between the spherical part of the couple-stress and the extra energy term observed in [7, 9], and (ii) is thus furnished with a theoretical mechanism/method that enables determination of the spherical part of the couple-stress outside the standard, well-known equilibrium conventions.

The present communication aims to provide some further information and better understanding of the manner in which this newly developed theoretical mechanism or method [12] is applied in specific boundary value problems met in polar linear elasticity of fibrous composites. In this context, it tests the applicability of the method in the case that a rectangular plate reinforced by a single family of unidirectional fibres is subjected to pure bending. In the special case that fibres are absent, this is recognised as the classical pure bending problem of non-polar isotropic elastic plates (e.g. [14]). The corresponding plane strain problem and its solution is also classical and older. For the case of material isotropy, this is detailed in classical texts as well $[14,15]$. Moreover, an anisotropic material generalisation of the latter has also been found useful in a recent relevant publication [16].

The three-dimensional boundary value problem of present interest is thus regarded as one of the simplest linear elasticity problems that can be considered in the fibrous composite plate literature. A proper and full description of this problem is detailed in Sect. 3, immediately after some necessary preliminary features of the aforementioned couple-stress theory generalisation [12] are briefly quoted in Sect. 2. Section 3 also provides the problem solution in the case of either non-polar or polar material behaviour, where the fibres are considered perfectly flexible or resistant in bending, respectively. It thus verifies the fact that the aforementioned antisymmetric rotation fields, $\omega$ and $\varphi$, are indeed different.

Section 4 next describes in detail the form attained by the couple-stress constitutive equation, including both its deviatoric and spherical parts, and by applying the implied newly developed method, demonstrates the manner that the latter part can be determined in linear polar elasticity of fibrous composites. It is recalled that there are currently available three versions of linear polar elasticity for fibrous composites, namely (i) the unrestricted theory and (ii) its restricted, bending mode version [7, 9], as well as (iii) a recently emerged restricted version $[12,17]$ that is principally associated with fibre-splay deformation features. As is shown in Sect. 4 and is also discussed afterwards as a part of the concluding discussion and observations detailed in Sect. 5, accuracy of the spherical couple-stress determination naturally depends on the extent to which the employed version of the theory sufficiently captures the principal deformation features of the considered boundary value problem.

\section{Generalised formulation of the couple-stress theory for linearly elastic solids}

Consider a suitable Cartesian coordinate framework $\mathrm{O} x_{i}$, where, here as well as in what follows, indices take the values 1,2, and 3, and, wherever necessary, the summation notation of repeated indices also applies. Quotation of the principal equations of the Cosserat couple-stress theory [13] may begin with the standard decomposition,

$$
\sigma_{i j}=\sigma_{(i j)}+\sigma_{[i j]}
$$

of the components of the non-symmetric stress tensor, $\sigma$, into symmetric and antisymmetric parts, and continue with the equilibrium equations

$$
\sigma_{i j, i}=0, \sigma_{[i j]}=\frac{1}{2} \varepsilon_{k j i} m_{\ell k, \ell},
$$

where $\boldsymbol{m}$ denotes the corresponding couple-stress tensor, $\boldsymbol{\varepsilon}$ represents the alternating tensor, and, for simplicity, body forces and body couples are neglected. 
In (2.1) and (2.2) the components of the tensors $\boldsymbol{\sigma}$ and $\boldsymbol{m}$ have been assumed differentiable functions of the implied coordinate parameters. Under the assumption that the derivatives of $\boldsymbol{m}$ appearing in (2.2.2) are also differentiable, a combination of (2.1) with the equilibrium Eqs. (2.2) leads to

$$
\sigma_{(i j), i}+\frac{1}{2} \varepsilon_{k j i} \bar{m}_{\ell k, \ell i}=0
$$

where

$$
\bar{m}_{\ell k}=m_{\ell k}-\frac{1}{3} m_{r r} \delta_{\ell k}
$$

is the deviatoric part of the couple-stress tensor, and the appearing Kronecker's delta represents the components of the unit matrix, $\boldsymbol{I}$. While no constitutive equations are provided or required at this point, the absence of the spherical part of the couple-stress, $m_{r r}$, from the equations of equilibrium (2.3) implies that it cannot be determined by use of the outlined standard equilibrium considerations.

Along with the above equations, the components of the traction and the couple-traction vectors acting on any internal or bounding surface of the material are, respectively, given as follows:

$$
T_{i}^{(n)}=\sigma_{j i} n_{j}, L_{i}^{(n)}=m_{j i} n_{j},
$$

where $\boldsymbol{n}$ denotes the outward unit normal of that surface. In the case of the bounding surface of an elastic solid, Eqs. (2.5) represent the traction and couple-traction boundary conditions applied externally on the material, respectively.

In line with the polar material extension of Clapeyron's theorem [16], it is postulated that, in the absence of body forces and body moments, the total energy stored within an arbitrary volume, $V$, of a polar linearly elastic material is

$$
E=\frac{1}{2} \int_{S}\left(T_{i}^{(n)} u_{i}+L_{i}^{(n)} \Phi_{i}\right) \mathrm{d} S \geq 0,
$$

where $S$ denotes the surface that surrounds $V, d S$ represents the corresponding surface element, and the equality sign holds only in the absence of deformation. Moreover, $\boldsymbol{u}$ (with components $u_{i}$ ) is the standard displacement vector that is conjugate to the traction vector $\boldsymbol{T}^{(n)}$, and $\boldsymbol{\Phi}$ (with components $\Phi_{i}$ ) is some appropriately specified spin-type vector that (i) characterises the couple-stress theory of interest, (ii) is generally dependent on the displacement gradients, and (iii) is conjugate to the couple-traction vector $\boldsymbol{L}^{(n)}$.

This characteristic spin vector, $\boldsymbol{\Phi}$, is perceived as the vector of a corresponding, antisymmetric, rotationtype tensor, $\varphi$, in the sense that

$$
\Phi_{i}=\frac{1}{2} \varepsilon_{i j k} \varphi_{k j}, \varphi_{k j}=\varepsilon_{i j k} \Phi_{i}
$$

It is recalled that the conventional/general spin vector of the implied deformation, $\boldsymbol{\Omega}$, and the antisymmetric rotation tensor, $\omega$, also obey these relationships, so that

$$
\Omega_{\mathrm{i}}=\frac{1}{2} \varepsilon_{i j k} \omega_{k j}, \omega_{k j}=\varepsilon_{i j k} \Omega_{i} .
$$

In this context, the outlined generalised couple-stress theory reduces naturally to its conventional Cosserat counterpart as soon as $\boldsymbol{\Phi}$ is considered identical to $\boldsymbol{\Omega}$ (or, equivalently, $\boldsymbol{\varphi} \equiv \boldsymbol{\omega}$ ) in what follows.

Regardless of the choice of $\boldsymbol{\Phi}$, a combination of (2.5) and (2.6), followed by application of the divergence theorem and the product rule of differentiation, leads to

$$
E=\frac{1}{2} \int_{V}\left[\sigma_{j i} u_{i, j}+\left(m_{j i} \Phi_{i}\right)_{, j}\right] \mathrm{d} V,
$$

where (2.2.1) is also taken into consideration. Moreover, due to the symmetry and the antisymmetry, respectively, of the standard small strain and rotation tensors,

$$
e_{i j}=\frac{1}{2}\left(u_{i, j}+u_{j, i}\right), \omega_{i j}=\frac{1}{2}\left(u_{i, j}-u_{j, i}\right),
$$


(2.9) is seen equivalent to the following:

$$
E=\frac{1}{2} \int_{V}\left[\sigma_{(j i)} e_{i j}+\sigma_{[j i]} \omega_{i j}+\left(m_{\ell i} \Phi_{i}\right)_{, \ell}\right] \mathrm{d} V
$$

Use of (2.2.2) then leads to

$$
E=\frac{1}{2} \int_{V}\left[\sigma_{(j i)} e_{i j}+\frac{1}{2} \varepsilon_{k i j} m_{\ell k, \ell} \omega_{i j}+\left(m_{\ell i} \Phi_{i}\right)_{, \ell}\right] \mathrm{d} V
$$

and, by further use of (2.8.2), one obtains

$$
E=\frac{1}{2} \int_{V}\left[\sigma_{(j i)} e_{i j}+m_{\ell i, \ell}\left(\Phi_{i}-\Omega_{i}\right)+m_{\ell i} \Phi_{i, \ell}\right] \mathrm{d} V \equiv \int_{V}\left(W^{e}+W^{\Phi}\right) \mathrm{d} V=\int_{V} W \mathrm{~d} V .
$$

The positive semidefinite quantities

$$
W^{e}=\frac{1}{2} \sigma_{(j i)} e_{i j} \geq 0, W^{\Phi}=\frac{1}{2}\left[m_{\ell i, \ell}\left(\Phi_{i}-\Omega_{i}\right)+m_{\ell i} \Phi_{i, \ell}\right] \geq 0
$$

thus, respectively, represent the standard strain energy function met in non-polar linear elasticity and its generalised spin-gradient counterpart that is due to the considered polar material response of the elastic solid of interest. The equality sign appearing in (2.13.1) or (2.13.2) applies only in the absence of strain or polar material response, respectively. In this manner, the internal energy stored in the material is guaranteed to also be positive semidefinite,

$$
W=W^{e}+W^{\Phi} \geq 0
$$

with the equality holding only in the complete absence of deformation.

The outlined generalised couple-stress theory may be found useful in any kind of a polar linear elasticity application in which the characteristic spin vector $\boldsymbol{\Phi}$ acquires some specific physical meaning. As is already mentioned though, its formation (see also [12]) is essentially motivated by the observation that identification of $\boldsymbol{\Phi}$ with the concept of an appropriate fibre spin vector can lead to the determination of the spherical part of the couple-stress, which otherwise remains indeterminate in polar elasticity of fibre-reinforced materials.

The correctness and effectiveness of this observation is verified in Sect. 4, with an example application that connects the outlined theoretical development with the pure bending problem of a rectangular fibre-reinforced plate subjected to terminal couples. Meanwhile, Sect. 3 introduces this linear elasticity boundary value problem and provides the principal details of both its non-polar and polar elasticity solutions.

\section{Pure bending of a rectangular plate reinforced by a single family of straight fibres}

In the aforementioned Cartesian coordinate system, $O x_{i}$, consider a rectangular linearly elastic plate whose dimensions are such that $\left|x_{1}\right| \leq L_{1},\left|x_{2}\right| \leq h / 2$ and $\left|x_{3}\right| \leq L_{3}$ (see also Fig. 1). At the edges $x_{1}= \pm L_{1}$, the plate is subjected to the externally applied normal stress distribution

$$
\sigma_{11}=\hat{\sigma}_{1} x_{2}
$$

where $\hat{\sigma}_{1}$ is a known positive constant (see Fig. 2). No other traction is applied externally on any of the six boundary planes. The plate thus bends under the action of a pair of terminal couples with magnitude

$$
M_{3}=\left.\int_{-h / 2}^{h / 2} \sigma_{11}\right|_{x_{1}= \pm L_{1}} x_{2} \mathrm{~d} x_{2}=\frac{h^{3}}{12} \hat{\sigma}_{1},
$$

per unit plate width. The magnitude of the total bending moment applied externally on each of those edges $\left(x_{1}\right.$ $= \pm L_{1}$ ) is evidently equal to $L_{1} M_{3}=L_{1} h^{3} \hat{\sigma}_{1} / 12$.

The history and the relatively simple solution that this classical boundary value problem attains in the case of isotropic, non-polar linear elasticity may be found in textbooks (e.g. $[14,15])$. It is recalled in this context 


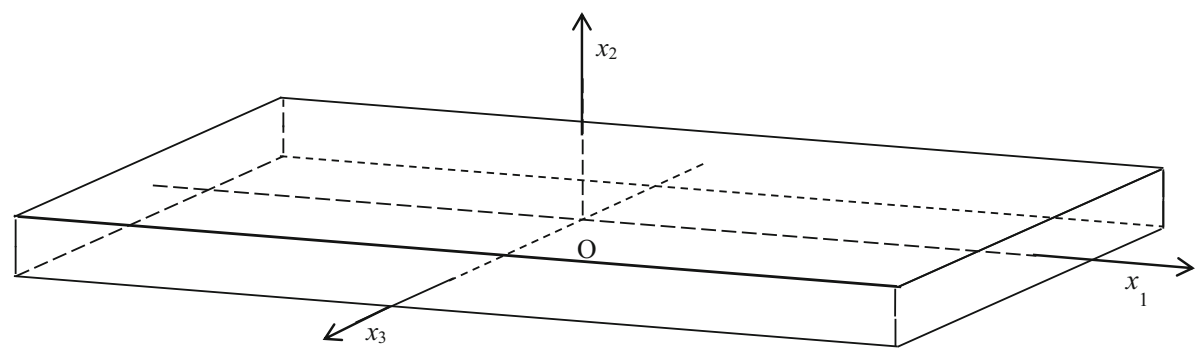

Fig. 1 Schematic representation of a rectangular plate in a suitably selected Cartesian coordinate system, $O x_{i}\left(-L_{1} \leq x_{1} \leq L_{1}\right.$, $\left.-h / 2 \leq x_{2} \leq h / 2,-L_{3} \leq x_{3} \leq L_{3}\right)$

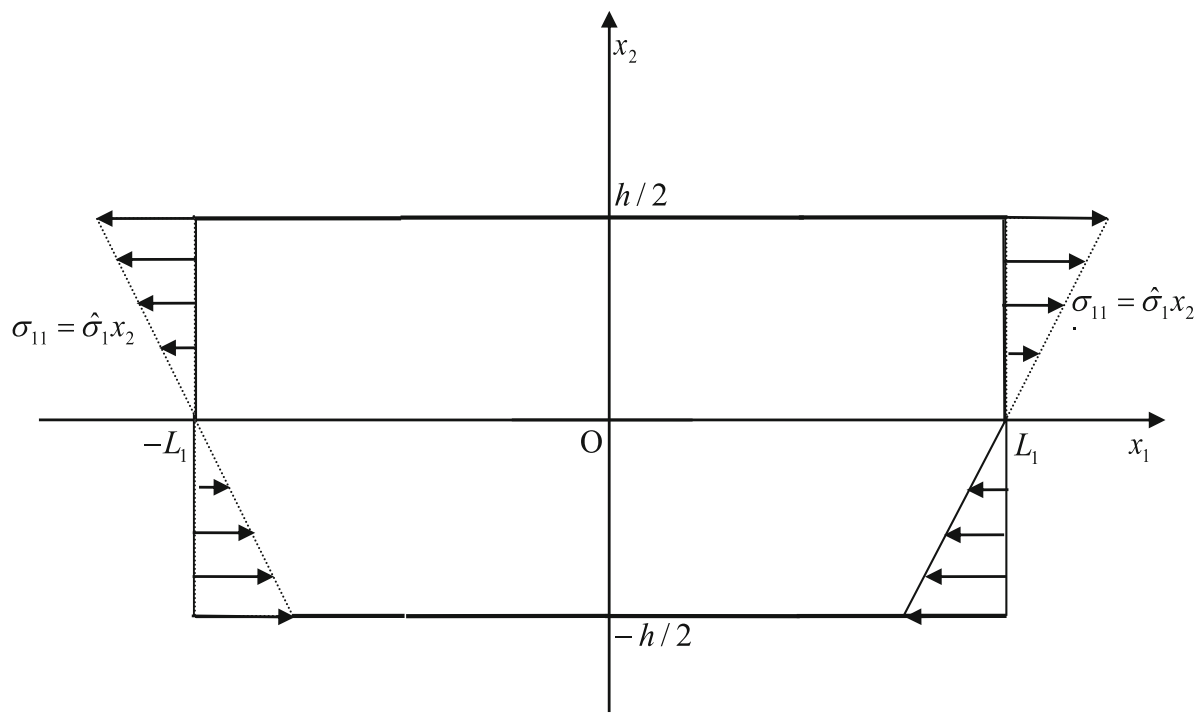

Fig. 2 Schematic representation of a plate cross section that is normal to the $x_{3}$-direction, featuring the boundary traction distributions which create pure bending in the case of non-polar linearly elastic material behaviour

that, in cases that the normal stress distribution applied externally at $x_{1}= \pm L_{1} / 2$ satisfies (3.2) but differs from (3.1), the implied isotropic elasticity solution is still considered accurate for sufficiently thin plates, by virtue of the Saint-Venant's principle.

In the present case of interest though, it is assumed that the plate has embedded a single family of straight fibres oriented along the $x_{1}$-direction. The fibres may be either perfectly flexible or resistant in bending and thus furnish the linearly elastic plate of interest with non-polar or polar transversely isotropic material characteristics, respectively. As is also mentioned in the Introduction, the corresponding plane strain problem is considered and solved in [16].

\subsection{Non-polar material behaviour}

The non-polar elasticity solution of the present three-dimensional version of this anisotropic linear elasticity problem may thus be considered as an appropriate, relatively simple extension of its counterparts detailed in the above publications. This begins with consideration of the stress-strain part of the relevant constitutive equation which, as the $x_{1}$-axis defines a preference material direction, is described as follows:

$$
\left[\begin{array}{l}
e_{11} \\
e_{22} \\
e_{33} \\
2 e_{23} \\
2 e_{13} \\
2 e_{12}
\end{array}\right]=\left[\begin{array}{llllll}
S_{11} & S_{12} & S_{12} & 0 & 0 & 0 \\
S_{12} & S_{22} & S_{12} & 0 & 0 & 0 \\
S_{12} & S_{12} & S_{22} & 0 & 0 & 0 \\
0 & 0 & 0 & S_{44} & 0 & 0 \\
0 & 0 & 0 & 0 & S_{66} & 0 \\
0 & 0 & 0 & 0 & 0 & S_{66}
\end{array}\right]\left[\begin{array}{l}
\sigma_{11} \\
\sigma_{22} \\
\sigma_{33} \\
\sigma_{(23)} \\
\sigma_{(13)} \\
\sigma_{(12)}
\end{array}\right],
$$


where the appearing $6 \times 6$ matrix of elastic compliances, $S$, is the inverse of the corresponding matrix of elastic stiffness, $\boldsymbol{C}$ (e.g. [18]). Moreover, the suffices associated with the shear stress components are enclosed in parentheses to signify that (3.3) will also still hold, as one of the constitutive equations met later in the description of the corresponding polar elasticity problem. A second constitutive equation that relates the couple-stresses and fibre curvature-strains is required in this case, and this will be introduced in Sect. 3.2, as appropriate.

It can readily be verified that if (3.1) and

$$
\sigma_{22}=\sigma_{33}=0, \quad \sigma_{12} \equiv \sigma_{(12)}=0, \quad \sigma_{13} \equiv \sigma_{(13)}=0, \quad \sigma_{23} \equiv \sigma_{(23)}=0, \quad \sigma_{[i j]} \equiv 0
$$

are all adopted to represent the stress distribution throughout the body of the plate, then the implied all-around traction boundary conditions and the corresponding equilibrium equations, namely (2.2) with $\boldsymbol{m} \equiv \mathbf{0}$, are satisfied identically. Thus, (3.1) and (3.4) represent the stress distribution associated with the unique, exact solution of the non-polar version of this anisotropic linear elasticity boundary value problem, provided that a corresponding displacement field does exist and is identified.

That displacement field becomes indeed available by inserting the stress field (3.1) and (3.4) into (3.3) and combining the resulting equations with the definition (2.10.1) of the strain tensor. In this manner, one obtains the following set of six simultaneous partial differential equations for the three unknown displacement components:

$$
\begin{aligned}
& u_{1,1}=S_{11} \hat{\sigma}_{1} x_{2}, u_{2,2}=S_{12} \hat{\sigma}_{1} x_{2}, u_{3,3}=S_{12} \hat{\sigma}_{1} x_{2}, \\
& u_{2,3}+u_{3,2}=0, u_{1,3}+u_{3,1}=0, u_{1,2}+u_{2,1}=0 .
\end{aligned}
$$

Integrating this set of equations in a manner that ensures strain compatibility, and ignoring rigid body translation, one obtains the displacement field sought as follows:

$$
u_{1}=\hat{\sigma}_{1} S_{11} x_{1} x_{2}, u_{2}=\frac{1}{2} \hat{\sigma}_{1}\left[S_{12}\left(x_{2}^{2}-x_{3}^{2}\right)-S_{11} x_{1}^{2}\right], u_{3}=\hat{\sigma}_{1} S_{12} x_{3} x_{2}
$$

where care is also taken for the rotations, $\omega_{i j}$, to vanish at the coordinate origin.

The outlined derivations suffice for the description and the solution of this simple boundary value problem in the case of non-polar linear elasticity, where fibres are considered perfectly flexible. Indeed, as $\boldsymbol{m} \equiv \mathbf{0}$, a combination of the displacement field (3.6) with the stress field (3.1) and (3.4) suffices to satisfy the equilibrium Eqs. (2.2) (or, equivalently, (2.3)) and all the externally applied boundary conditions.

\subsection{Polar material behaviour}

However, in the polar linear elasticity case, some further investigation is required on whether (i) the equilibrium equations as well as the relevant set of traction boundary conditions are still satisfied, and (ii) a corresponding set of homogeneous couple-traction boundary conditions suffices to maintain the displacement field (3.6). Moreover, as polar material behaviour is here attributed to the resistance that individual fibres exhibit in specific deformation modes, a further investigation is required on the extent to which (iii) the method proposed in [12], in conjunction with the generalised couple-stress theory briefed in Sect. 2, enables the determination of the spherical part of the couple-stress.

In search for definite answers to these questions, the subsequent section will introduce and handle separately each one of all three of the existing versions of polar linear elasticity for fibrous composites [7, 9, 17]. Nevertheless, it may already be noticed that the quadratic form of the displacement components (3.6) guarantees a positive answer to the first of the above questions. This is because the curvature-strains employed in any of the implied versions of the theory [7, 9, 17] are second-order spatial derivatives of the displacements, and, in the present problem, they will all emerge constant (see also Sect. 4).

The couple-stresses obtained by use of any of the three available relevant sets of linear constitutive equations will thus also be constant, and (2.2.2) will thus yield

$$
\sigma_{[i j]}=0 .
$$

This equation, which agrees with and, in fact, replaces the corresponding non-polar elasticity identity (3.4.5), makes then evident that the anticipated stress field will still satisfy the equations of equilibrium as well as all the traction boundary conditions applied all-around the plate boundaries. 


\subsection{Connection of the polar elasticity problem with the generalised couple-stress theory}

On the other hand, and in close connection with (2.14), the strain energy function of polar, linearly elastic fibrous composites is of the form

$$
W=W^{e}+W^{k} \geq 0,
$$

where $W^{e}$ is the strain energy function underpinning the non-polar solution outlined already in Sect. 3.1. Moreover, $W^{k}$ emerges by consideration of deformation effects associated with the fibre direction gradients and, necessarily, should thus be identical with the spin energy involved in the strain energy function (2.14) of the generalised couple-stress theory $\left(W^{\Phi} \equiv W^{k}\right)$.

Most importantly, $W^{k}$ contains an extra energy term which, as already noted in the Introduction and explained further in Sect. 4, does not contribute to the couple-stress constitutive equation. As this term thus leaves unaffected the state of equilibrium, its emergence in $W^{k}$ is necessarily interpreted as energy contribution that is due to the action of the spherical part of the couple-stress, which does not affect equilibrium either. A comparison is then required between the expressions of $W^{k}$ and $W^{\Phi}$, the former of which is explicitly provided in any of the three available versions of the polar linear elasticity for fibrous composites (see Sect. 4). Nevertheless, (2.13.2) reveals that evaluation of $W^{\Phi}$ requires prior determination of the spin vectors $\boldsymbol{\Omega}$ and $\boldsymbol{\Phi}$.

It is accordingly noted that the already determined displacement field (3.6) gives rise to the following deformation spin vector:

$$
\boldsymbol{\Omega}=\left(\omega_{32}, \omega_{13}, \omega_{21}\right)^{T}=\left(\hat{\sigma}_{1} S_{12} x_{3}, 0,-\hat{\sigma}_{1} S_{11} x_{1}\right)^{T},
$$

and yields the tangent vector of the deformed fibres as follows:

$$
\boldsymbol{b}=\boldsymbol{a}+\boldsymbol{u}_{, 1}=\left(1+\hat{\sigma}_{1} S_{11} x_{2}, \quad-\hat{\sigma}_{1} S_{11} x_{1}, 0\right)^{T},
$$

where

$$
\boldsymbol{a}=(1,0,0)^{T}
$$

is the direction vector of the undeformed fibres.

On the other hand, the fibre spin vector, $\boldsymbol{\Phi}$, which characterises the generalised couple-stress theory of present interest is defined as follows [12]:

$$
\Phi=\tilde{\phi} \tilde{\Phi}
$$

where

$$
\tilde{\Phi}=\boldsymbol{a} \times \boldsymbol{b}=\left(0,0, \quad-\hat{\sigma}_{1} S_{11} x_{1}\right)^{T},
$$

and the scalar quantity $\tilde{\phi}$ is to be determined. This result shows that $\boldsymbol{\Phi}$ and $\boldsymbol{\Omega}$ have different directions and, as anticipated in [12] (see also Sect. 2), verifies that these are therefore different spin vectors.

Connection of (3.12) with (2.13.2) enables next the spin energy of the generalised couple-stress theory to be rearranged as follows:

$$
\begin{aligned}
W^{\Phi} & =\frac{1}{2}\left[\left(\frac{1}{3} m_{r r, \ell} \delta_{\ell i}+\bar{m}_{\ell i, \ell}\right)\left(\tilde{\phi} \tilde{\Phi}_{i}-\Omega_{i}\right)+\left(\frac{1}{3} m_{r r} \delta_{\ell i}+\bar{m}_{\ell i}\right)\left(\tilde{\phi} \tilde{\Phi}_{i}\right)_{, \ell}\right] \\
& =W_{1}^{\Phi}\left(m_{r r}, \tilde{\phi}\right)+W_{2}^{\Phi}\left(\bar{m}_{\ell i}, \tilde{\phi}\right),
\end{aligned}
$$

where

$$
W_{1}^{\Phi}\left(m_{r r}, \tilde{\phi}\right)=\frac{1}{6}\left[\left(m_{r r} \tilde{\phi} \tilde{\Phi}_{i}\right)_{, i}-m_{r r, i} \Omega_{i}\right], W_{2}^{\Phi}\left(\bar{m}_{\ell i}, \tilde{\phi}\right)=\frac{1}{2}\left[\left(\bar{m}_{\ell i} \tilde{\phi} \tilde{\Phi}_{i}\right)_{, \ell}-\bar{m}_{\ell i, \ell} \Omega_{i}\right]
$$

depend on the spherical part and on the components of the deviatoric part of the couple-stress tensor, respectively. With use of (3.9) and (3.13), these quantities thus obtain more specific forms, namely

$$
W_{1}^{\Phi}\left(m_{r r}, \tilde{\phi}\right)=-\frac{\hat{\sigma}_{1}}{6}\left[S_{11}\left(x_{1} \tilde{\phi} m_{r r}\right)_{, 3}+S_{12} x_{3} m_{r r, 1}-S_{11} x_{1} m_{r r, 3}\right],
$$




$$
W_{2}^{\Phi}\left(\bar{m}_{\ell i}, \tilde{\phi}\right)=-\frac{\hat{\sigma}_{1}}{2}\left[S_{11}\left(x_{1} \bar{m}_{\ell 3} \tilde{\phi}\right)_{, \ell}+S_{12} x_{3} \bar{m}_{\ell 1, \ell}-S_{11} x_{1} \bar{m}_{\ell 3, \ell}\right],
$$

which comply with the specific features of the plate bending problem of present interest.

The aforementioned comparison that is required between $W^{\Phi}$ and $W^{k}$ can then be performed as soon as a specific version of the polar linear elasticity for fibrous composites is selected and, hence, the corresponding form of $W^{k}$ is explicitly specified. The relevant process is detailed in the subsequent Section, where attention is paid to all three of the existing linear versions of the theory. In line with the relevant developments detailed in $[12,17]$, Sect. 4 thus begins with the simplest and ends with the most complicated version of the theory.

\section{Couple-stress field and determination of its spherical part}

4.1 The fibre-bending deformation mode/version of the theory

The curvature-strain part of the strain energy function employed in the restricted, fibre-bending version of the theory is as follows [7, 9]:

$$
W^{K}=\frac{3}{8} d^{f} K_{j} K_{j}+\bar{\gamma}\left(a_{j} K_{j}\right)^{2}=\frac{3}{8} d^{f}\left(K_{1}^{2}+K_{2}^{2}+K_{3}^{2}\right)+\bar{\gamma} K_{1}^{2}, K_{i}=u_{i, k j} a_{k} a_{j}=u_{i, 11},
$$

where $K_{i}$ is the relevant fibre curvature vector and (3.11) still holds. Moreover, $d^{f}$ represents the single fibrebending stiffness that enters actively the constitutive equation of the deviatoric part of the couple-stress tensor, namely

$$
\bar{m}_{\ell r}=d^{f} \varepsilon_{r s i} K_{i} a_{\ell} a_{s}=d^{f} \varepsilon_{r 1 i} K_{i} a_{\ell}=d^{f} \varepsilon_{r 1 i} u_{i, 11} a_{\ell},
$$

while the additional relevant modulus, $\bar{\gamma}$, enters the extra energy term that leaves unaffected both the constitutive Eq. (4.2) and the state of equilibrium. Evidently, positive definiteness of (4.1) requires

$$
d^{f}>0, \quad \bar{\gamma}>0 .
$$

A combination of (4.1.2) with (3.6) then shows that the only nonzero curvature-strain component is

$$
K_{2}=u_{2,11}=-\hat{\sigma}_{1} S_{11} .
$$

Hence, in this plate bending problem, the curvature part of the strain energy function is

$$
W^{K}=\frac{3}{8} d^{f} K_{2}^{2}=\frac{3}{8} d^{f}\left(\hat{\sigma}_{1} S_{11}\right)^{2},
$$

and, in a similar manner, the only nonzero component of the deviatoric part of the stress tensor is

$$
\bar{m}_{13}=d^{f} K_{2}=-d^{f} \hat{\sigma}_{1} S_{11} \text {. }
$$

This version of the theory thus predicts that the deviatoric part of every normal couple-stress is zero $\left(\bar{m}_{11}=\bar{m}_{22}=\bar{m}_{33}=0\right)$, and (2.4) then yields

$$
m_{11}=m_{22}=m_{33}=\frac{1}{3} m_{r r}
$$

These results make it clear that, in line with the corresponding plane strain problem [16], the implied pure bending deformation of the fibre-reinforced rectangular plate is sustainable within the framework of the fibrebending deformation version of polar linear elasticity, only if, in addition to the traction boundary conditions (2.1), a couple-traction having constant magnitude

$$
\hat{m}_{3}=\left|\bar{m}_{13}\right|=d^{f} \hat{\sigma}_{1} S_{11}
$$

and direction parallel to the $x_{3}$-axis is also applied externally on the boundaries $x_{1}= \pm L_{1}$, in a point-by-point sense (see Fig. 3 for a schematic representation). Further relevant explanations, comments, and discussion may thus be found in [16] and will not be repeated here. 


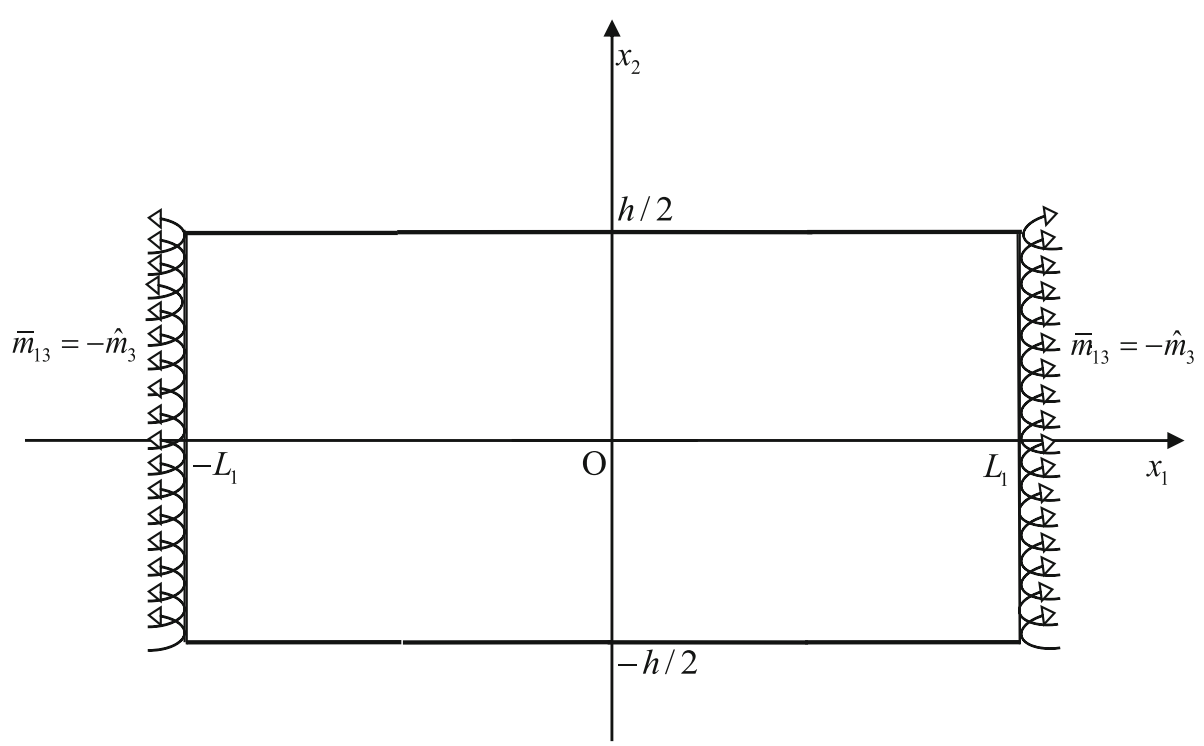

Fig. 3 Schematic representation of constant couple-traction distributions superposed on the externally applied loading depicted in Fig. 2, for a corresponding polar fibre-reinforced elastic rectangular plate to maintain the pure bending deformation (3.6)

It is important to notice at this point that, due to the simplicity of the displacement field (3.6), (4.1.2) returns $K_{1}=0$, and, in this boundary value problem, the extra energy term appearing in (4.1.1) thus attains a zero value. This fact though does not prevent the present analysis to proceed and, by setting equal to zero the extra energy term (3.16.1) that emerges in the generalised couple-stress theory, to determine the spherical part of the couple-stress and its distribution throughout the fibrous composite plate of interest.

Accordingly, by requiring equality of the polar elasticity parts (3.14) and (4.5) of the strain energy function encountered in the generalised couple-stress theory and the present theory, respectively, one obtains

$$
W_{1}^{\Phi}\left(m_{r r}, \tilde{\phi}\right)=0, W_{2}^{\Phi}\left(\bar{m}_{\ell i}, \tilde{\phi}\right)=\frac{3}{8} d^{f}\left(\hat{\sigma}_{1} S_{11}\right)^{2} .
$$

The first of these equations implies that this is an exceptional boundary value problem, in which the action of the spherical part of the couple-stress does not store work in the material of the deformed plate. With use of (3.16) and (4.6), (4.9) are then transformed into the following pair of simultaneous partial differential equations (PDEs):

$$
\left(x_{1} \tilde{\phi} m_{r r}\right)_{, 3}+\left(S_{12} / S_{11}\right) x_{3} m_{r r, 1}-x_{1} m_{r r, 3}=0,\left(x_{1} \tilde{\phi}\right)_{, 1}=\frac{3}{4}
$$

for the pair of the unknown scalars $m_{r r}$ and $\tilde{\phi}$.

Solution to the second of these PDEs yields

$$
\tilde{\phi}=\frac{3}{4}+\frac{1}{x_{1}} f\left(x_{2}, x_{3}\right)
$$

where $f$ is an arbitrary function of its arguments. However, the fibre spin vector, $\boldsymbol{\Phi}$, is anticipated finite throughout the plate volume, including the internal plane $x_{1}=0$, and this is possible only if $f=0$. It follows that

$$
\tilde{\phi}=3 / 4, \Phi=\left(0,0,-3 \hat{\sigma}_{1} S_{11} x_{1} / 4\right)^{T},
$$

where use is also made of (3.12) and (3.13).

With the value of the parameter $\tilde{\phi}$ being thus determined, the PDE (4.10.1) simplifies into the following:

$$
4\left(S_{12} / S_{11}\right) x_{3} m_{r r, 1}-x_{1} m_{r r, 3}=0 .
$$


A general solution to this PDE is obtained in the Appendix with use of the method of characteristic lines, and is as follows:

$$
m_{r r}=m_{r r}\left(x_{2}, u\right), u=\frac{x_{1}^{2}}{4 S_{12}}+\frac{x_{3}^{2}}{S_{11}} .
$$

This reveals that the implied characteristic lines are ellipses that lie on the $x_{1} x_{3}$-plane and that the magnitude of their axes depends only on the elastic moduli of the non-polar elastic plate.

A less general and, hence, more specific solution of (4.12), may be obtained with use of the method of the separation of variables (see the Appendix). This is evidently a special case of (4.13) and is of the form

$$
m_{r r}=f_{2}\left(x_{2}\right) \exp \left\{g_{2}\left(x_{2}\right)\left(\frac{x_{1}^{2}}{4 S_{12}}+\frac{x_{3}^{2}}{S_{11}}\right)\right\}
$$

where $f_{2}\left(x_{2}\right)$ and $g_{2}\left(x_{2}\right)$ are arbitrary functions of the thickness coordinate parameter, $x_{2}$. It is observed that some more specific form of either (4.13) or (4.14) may be sought as soon as a set of relevant boundary conditions is associated with any of the normal couple-stresses (4.7).

If, for instance, it is assumed that $m_{22}=0$ on the top and bottom planes of the plate $\left(x_{2}= \pm h / 2\right)$ then, any choice of the form

$$
f_{2}=f_{2}\left(x_{2}^{2}-h^{2} / 4\right)
$$

is qualified as admissible to enter (4.14). In that case though, the displacement field (3.6) is sustainable within the framework of the present version of the theory, only if, in line with (4.7), the following additional boundary conditions are also satisfied on the remaining plate boundaries:

$$
\begin{aligned}
& \left.m_{11}\right|_{x_{1}= \pm L_{1}}=f_{2}\left(x_{2}^{2}-h^{2} / 4\right) \exp \left\{g_{2}\left(x_{2}\right)\left(\frac{L_{1}^{2}}{4 S_{12}}+\frac{x_{3}^{2}}{S_{11}}\right)\right\}, \\
& \left.m_{33}\right|_{x_{3}= \pm L_{3}}=f_{2}\left(x_{2}^{2}-h^{2} / 4\right) \exp \left\{g_{2}\left(x_{2}\right)\left(\frac{x_{1}^{2}}{4 S_{12}}+\frac{L_{3}^{2}}{S_{11}}\right)\right\} .
\end{aligned}
$$

In this regard, it becomes evident that, in the present boundary value problem, the specific form of the arbitrary functions $f_{2}\left(x_{2}\right)$ and $g_{2}\left(x_{2}\right)$ is essentially dictated by the known form of the externally applied normal boundary couple-tractions.

It is then also of interest to note that the choice $f_{2}\left(x_{2}\right)=0$ makes the displacement field (3.6) attainable in the complete absence of normal boundary couple-tractions. In other words, if no normal couple-stresses are applied externally on any of the plate boundaries, then the choice (4.14) predicts that, in this boundary value problem, all three normal couple-stresses are zero throughout the body of the plate.

\subsection{The fibre-splay deformation mode/version of the theory}

Like the unrestricted version of the theory, which is considered separately in Sect. 4.3, its restricted, fibre-splay version makes use of the full form of the fibre curvature-strain tensor, namely

$$
\kappa_{i j}=\left(u_{i, k} a_{k}\right)_{, j}=u_{i, j 1},
$$

where (3.11) has evidently again been used. However, this version of the theory imposes the following restriction on the curvature-strain part of the strain energy function [17]:

$$
W^{\kappa}\left(\boldsymbol{\kappa}_{s}, \boldsymbol{\kappa}_{a}, \boldsymbol{a}\right)=W^{\kappa}\left(\boldsymbol{\kappa}_{a}, \boldsymbol{\kappa}_{s}, \boldsymbol{a}\right)=W^{\kappa}(\boldsymbol{\kappa}, \boldsymbol{a}),
$$

where the components of the symmetric, $\kappa_{\mathbf{s}}$, and the antisymmetric, $\boldsymbol{\kappa}_{\boldsymbol{a}}$, parts of $\boldsymbol{\kappa}$ are, respectively,

$$
\kappa_{(i j)}=\frac{1}{2}\left(\kappa_{i j}+\kappa_{j i}\right)=\frac{1}{2}\left(u_{i, j 1}+u_{j, i 1}\right), \quad \kappa_{[i j]}=\frac{1}{2}\left(\kappa_{i j}-\kappa_{j i}\right)=\frac{1}{2}\left(u_{i, j 1}-u_{j, i 1}\right) .
$$

The restriction (4.18) requires from $W^{\kappa}$ to acquire the form

$$
W^{k}=\beta_{1}\left(\kappa_{(n n)}\right)^{2}+\beta_{2} \kappa_{(n n)} a_{k} \kappa_{(k m)} a_{m}+\hat{\beta}_{3}\left(a_{k} \kappa_{(k m)} a_{m}\right)^{2}
$$




$$
=\beta_{1}\left(\kappa_{n n}\right)^{2}+\beta_{2} \kappa_{n n} a_{k} \kappa_{k m} a_{m}+\hat{\beta}_{3}\left(a_{k} \kappa_{k m} a_{m}\right)^{2},
$$

and, hence, to make use of three elastic moduli, whose values are required to obey the inequalities

$$
\beta_{1} \geq 0, \beta_{2}+\hat{\beta}_{3} \geq 0, \hat{\beta}_{3} \geq \beta_{2}^{2} / 4 \beta_{1} .
$$

It is recalled that $\beta_{1}$ and $\beta_{2}$ are here elastic moduli that participate actively in the constitutive equation of the deviatoric the couple-stress, namely

$$
\bar{m}_{\ell r}=\frac{2}{3} \varepsilon_{r \ell s} a_{s}\left(2 \beta_{1} \kappa_{n n}+\beta_{2} \kappa_{k m} a_{k} a_{m}\right)=\frac{2}{3} \varepsilon_{r \ell s} a_{s}\left(2 \beta_{1} \kappa_{(n n)}+\beta_{2} \kappa_{(k m)} a_{k} a_{m}\right),
$$

while $\hat{\beta}_{3}$ regulates the extra energy term that leaves unaffected both (4.22) and the state of equilibrium.

In the present problem of interest, the displacement field (3.6) produces only two nonzero curvature-strain components, namely

$$
\kappa_{12}=u_{1,21}=\hat{\sigma}_{1} S_{11}, \quad \kappa_{21}=u_{2,11}=-\hat{\sigma}_{1} S_{11} .
$$

The symmetric part of the curvature-strain tensor thus is identically zero $\left(\kappa_{(i j)}=0\right)$, while its antisymmetric part contains only a single pair of nonzero components, namely

$$
\kappa_{[12]}=-\kappa_{[21]}=\hat{\sigma}_{1} S_{11} .
$$

Introduction of these results to (4.20) then yields

$$
W^{k}=\beta_{1}\left(\kappa_{n n}\right)^{2}+\beta_{2} \kappa_{n n} \kappa_{11}+\hat{\beta}_{3} \hat{\kappa}_{11}^{2}=0,
$$

which essentially reveals that fibre-splay deformation does not contribute to the strain energy function at all.

This rather interesting result should not come as a surprise, because the present problem of interest is a classical, pure bending problem that involves no other types of plate deformation. In other words, no fibre-splay deformation takes place, and, as the bending and the splay deformation modes are totally uncoupled in this pure bending problem, a combination of (4.22) and (4.23) yields

$$
\bar{m}_{\ell r}=0 \text {. }
$$

As far as this example application is concerned, the fibre-splay deformation version of the polar theory simply coincides with the non-polar theory of linear elasticity.

\subsection{The unrestricted theory}

The unrestricted theory [7, 9] accounts for full coupling of fibre-bending, fibre-splay, and fibre-twist deformation effects and is thus considerably more complicated than either of its restricted fibre-bending and fibre-splay versions. Like its fibre-splay counterpart, this makes use of the full form of the fibre curvature-strain tensor (4.17) as well as of the curvature-strain decomposition (4.19) but makes no use of the restriction (4.18).

Instead, this full version of the theory relies on the following, complete form of the polar part of strain energy function:

$$
W^{\kappa}=\beta_{1} J_{1}^{2}+\beta_{2} J_{1} J_{2}+\hat{\beta}_{3} J_{2}^{2}+\beta_{3} J_{3}+\beta_{4} J_{4}+\beta_{5} J_{5}+\beta_{6} J_{6}+\beta_{7} J_{7}
$$

where the appearing deformation invariants are

$$
J_{1}=\operatorname{tr} \kappa_{s}=\operatorname{tr} \kappa, J_{2}=\mathbf{a} \kappa_{s} \mathbf{a}=\mathbf{a} \kappa \mathbf{a}, J_{3}=\operatorname{tr} \kappa_{s}^{2}, J_{4}=\mathbf{a} \kappa_{s}^{2} \mathbf{a}, \quad J_{5}=\operatorname{tr} \kappa_{a}^{2}, J_{6}=\mathbf{a} \kappa_{a}^{2} \mathbf{a}, \quad J_{7}=\mathbf{a} \kappa_{s} \kappa_{a} \mathbf{a} .
$$

As is also noted in [12], the first three of the terms appearing at the right-hand side of (4.27) correspond to their counterparts employed earlier in (4.20). It follows that $\hat{\beta}_{3} J_{2}^{2}$ is still the extra energy term that does not affect the state of equilibrium. This becomes further clear from the absence of $\hat{\beta}_{3}$ in the corresponding constitutive equation of the deviatoric couple-stress which, for straight fibres parallel to the $x_{1}$-direction, is as follows:

$$
\bar{m}_{\ell r}=\frac{2}{3} \varepsilon_{r \ell 1}\left(2 \beta_{1} \kappa_{n n}+\beta_{2} \kappa_{11}\right)+\frac{2}{3} \varepsilon_{r i 1}\left(2 \beta_{3} \kappa_{(i \ell)}+\beta_{4} a_{\ell} \kappa_{(i 1)}\right)
$$




$$
-\frac{1}{3} \varepsilon_{r i s}\left\{4 \beta_{5}\left(a_{s} \kappa_{[i \ell]}+a_{\ell} \kappa_{[i s]}\right)+2 \beta_{6} a_{\ell}\left(a_{i} \kappa_{[s 1]}-2 a_{s} \kappa_{[i 1]}\right)+\beta_{7} a_{\ell}\left(a_{i} \kappa_{1 s}-2 a_{s} \kappa_{i 1}\right)\right\} .
$$

An alternative, explicit and practically more useful form of all nonzero components of this tensor is given as follows $[7,9,12]$ :

$$
\begin{aligned}
& {\left[\begin{array}{l}
\bar{m}_{11} \\
\bar{m}_{22} \\
\bar{m}_{33}
\end{array}\right]=\left[\begin{array}{ll}
0 & 2 b_{3} \\
-b_{2} & -b_{3} \\
b_{2} & -b_{3}
\end{array}\right]\left[\begin{array}{l}
e_{23,1} \\
\Omega_{1,1}
\end{array}\right],} \\
& {\left[\begin{array}{c}
-\bar{m}_{32} \\
\bar{m}_{23} \\
-\bar{m}_{12} \\
\bar{m}_{13}
\end{array}\right]=\left[\begin{array}{lllllll}
d_{11} & d_{22} & d_{33} & 0 & 0 & 0 & 0 \\
d_{11} & d_{33} & d_{22} & 0 & 0 & 0 & 0 \\
0 & 0 & 0 & d_{23} & d_{32} & 0 & 0 \\
0 & 0 & 0 & 0 & 0 & d_{23} & \hat{d}_{32}
\end{array}\right]\left[\begin{array}{l}
e_{11,1} \\
e_{22,1} \\
e_{33,1} \\
e_{31,1} \\
\Omega_{2,1} \\
e_{12,1} \\
\Omega_{3,1}
\end{array}\right],}
\end{aligned}
$$

where the appearing elastic moduli connect with their counterparts involved in (4.27) by the relations

$$
\begin{aligned}
& b_{2}=\frac{4}{3} \beta_{3}, \quad b_{3}=\frac{4}{3} \beta_{5}, \quad d_{11}=\frac{2}{3}\left(2 \beta_{1}+\beta_{2}\right), \quad d_{22}=\frac{4}{3}\left(\beta_{1}+\beta_{3}\right), \quad d_{33}=\frac{4}{3} \beta_{1}, \\
& d_{23}=\frac{1}{3}\left(4 \beta_{3}+2 \beta_{4}+3 \beta_{7}\right), \quad \hat{d}_{32}=-d_{32}=\frac{1}{3}\left(2 \beta_{4}+\beta_{7}-12 \beta_{5}-6 \beta_{6}\right) .
\end{aligned}
$$

These elastic moduli thus contain inherited information of the ability of the model to account not only for fibre-bending, but also for fibre-splay and fibre-twist types of deformation.

With the strain, the rotation, and the curvature-strain components of the pure bending problem of interest being already determined by (3.5), (3.9), and (4.19), respectively, it is next seen that the only nonzero deviatoric couple-stress stemming from (4.30) is

$$
\bar{m}_{13}=\hat{d}_{32} \Omega_{3,1}=-\hat{d}_{32} \hat{\sigma}_{1} S_{11} .
$$

It is recalled that (4.30.1) implies that the normal components of the deviatoric part of the couple-stress tensor may generally be nonzero. However, as $\bar{m}_{r r}=0$, the spherical part, $m_{r r}$, of the couple-stress tensor is still unspecified.

On the other hand, as (4.24) are still the only nonzero components of the antisymmetric part of the curvature-strain tensor while $\kappa_{(i j)}=0$, a combination of (4.27) and (4.28) reveals that

$$
W^{\kappa}=\beta_{5} J_{5}+\beta_{6} J_{6}=-\left(2 \beta_{5}+\beta_{6}\right) \kappa_{[12]}^{2}=D_{77}\left(\hat{\sigma}_{1} S_{11}\right)^{2},
$$

where the notion

$$
D_{77}=-\left(2 \beta_{5}+\beta_{6}\right)>0
$$

is employed to show that (4.33) agrees with its alternative form introduced in [9]. For the plate bending problem of present interest, this alternative form of $W^{\kappa}$ can in fact be expressed as follows [16]:

$$
W^{\kappa}=W^{\Omega}=D_{77} \Omega_{3,1}^{2} .
$$

A comparison of (4.32) and (4.33) with (4.6) and (4.5), respectively, shows a close resemblance of the present formulation with its counterpart outlined in Sect. 4.1 by use of the restricted, bending mode version of the theory. Indeed, the only relevant mathematical difference observed is essentially the fact that the two different active elastic moduli $\hat{d}_{32}$ and $D_{77}$ appearing at present in the constitutive Eq. (4.32) and the curvature part of the strain energy (4.33), respectively, are both replaced in the earlier formulation (Sect. 4.1) by the single active elastic modulus $d^{f}$. Nevertheless, the physical difference between the implied pair of different formulations is underpinned by the fact that, while $d^{f}$ emerged as a single elastic modulus of fibre-bending stiffness, either of $\hat{d}_{32}$ and $D_{77}$ emerges by suitable combination of coefficients that enable the general energy 
expression (4.27) to be regarded as a quadratic invariant representation of all three, fibre-bending, fibre-splay, and fibre-twist deformation modes.

It is thus noted that the unrestricted version of the theory still predicts that the deviatoric part of all three normal couple-stresses is zero $\left(\bar{m}_{11}=\bar{m}_{22}=\bar{m}_{33}=0\right)$ and, hence, that (4.7) still holds. Moreover, the implied pure bending deformation of the plate is still sustainable only if, in addition to the traction boundary conditions (2.1), the constant couple-traction depicted in Fig. 3 is also applied externally on the boundaries, $x_{1}$ $= \pm L_{1}$. However, (4.32) now implies that the magnitude of this boundary couple-traction is more accurately given according to

$$
\hat{m}_{3}=\hat{d}_{32} \hat{\sigma}_{1} S_{11}
$$

rather than to (4.8).

As, on the other hand, the displacement field (3.6) returns $\boldsymbol{\kappa}_{s}=\mathbf{0}$, the extra energy term $\hat{\beta}_{3} J_{2}^{2}$ appearing in (4.27) still attains a zero value. The equality requirement imposed between (4.35) and its polar counterpart (3.14) encountered in the generalised couple-stress theory thus leaves (4.9.1) unaltered but replaces (4.9.2) with

$$
W_{2}^{\Phi}\left(\bar{m}_{\ell i}, \tilde{\phi}\right)=D_{77}\left(\hat{\sigma}_{1} S_{11}\right)^{2} .
$$

Hence, with use of (3.16) and (4.32), (4.9.1) leads again to (4.10.1) while (4.37) yields

$$
\left(x_{1} \tilde{\phi}\right)_{, 1}=2 D_{77} / \hat{d}_{32} \text {. }
$$

It is accordingly observed that, as far as the present pure bending plate problem is concerned, the analytical simplification offered by the bending mode version of the theory considered in Sect. 4.1 is essentially confined in the approximation

$$
D_{77} / \hat{d}_{32}=3 / 8
$$

A treatment of (4.38) with the same solution process applied earlier on (4.19.2) then yields

$$
\tilde{\phi}=2 D_{77} / \hat{d}_{32}, \Phi=\left(0,0,-2 \hat{\sigma}_{1} S_{11} D_{77} x_{1} / \hat{d}_{32}\right)^{T} .
$$

This result enables (4.10.1) to replace (4.12) with the following PDE:

$$
\frac{\left(S_{12} / S_{11}\right)}{1-\left(2 D_{77} / \hat{d}_{32}\right)} x_{3} m_{r r, 1}-x_{1} m_{r r, 3}=0 \text {. }
$$

As a matter of fact, connection of this PDE with the fibre-bending mode approximation (4.39) leads naturally to (4.12).

The method of characteristic lines, employed in the Appendix for the solution of (4.12) then yields the general solution of (4.41) in the following form:

$$
m_{r r}=m_{r r}\left(x_{2}, v\right), \text { where }\left\{\begin{array}{l}
v=\frac{x_{1}^{2}}{4 S_{12} \hat{d}_{32}}+\frac{x_{3}^{2}}{S_{11}\left(\hat{d}_{32}-2 D_{77}\right)}, \text { if } \hat{d}_{32}>2 D_{77}, \\
v=\frac{x_{1}^{2}}{4 S_{12} \hat{d}_{32}}-\frac{x_{3}^{2}}{S_{11}\left|\hat{d}_{32}-2 D_{77}\right|}, \text { if } \hat{d}_{32}<2 D_{77},
\end{array}\right.
$$

which is essentially an appropriate generalisation of (4.13). This solution reveals that, dependent on the sign of the term $\hat{d}_{32}-2 D_{77}$, the implied characteristic lines are either ellipses or hyperbolas that lie on the $x_{1} x_{3}$-plane. However, the generality of the unrestricted theory now reveals further that the magnitude of the axes of those curves depends not only on the elastic moduli of the corresponding non-polar elastic plate but also on the micromechanics elastic moduli that characterise the deformation resistance of individual fibres.

In the rare and rather unlikely special case that $\hat{d}_{32}=2 D_{77},(4.41)$ predicts that $m_{r r}$ is essentially an arbitrary function of the coordinate parameters $x_{2}$ and $x_{3}$. If/when necessary, more specific forms of (4.42), like, for instance, forms that resemble (4.14), may also be obtained with use of the method of the separation of variables (see the Appendix). As is already mentioned towards the end of Sect. 4.1, determination of arbitrary functions of integration that enter any of the derived or implied solutions of (4.41) may be sought and obtained as soon as some specific set of boundary conditions is associated with the normal couple-stresses (4.7). 


\section{Further discussion and conclusions}

The pure bending problem of a rectangular fibrous composite plate, introduced and solved in Sects. 3 and 4 , is one of the simplest boundary value problems that verify the validity of the argument that underpins the generalised couple-stress theory briefed earlier in Sect. 2 (see also [12]). Namely, that the deformation spin vector, $\boldsymbol{\Omega}$, and its fibre spin counterpart, $\boldsymbol{\Phi}$, are in general different vectors. Based on the relevant arguments detailed in the Introduction, this verification favours the claim that the extra energy term that emerges in the strain energy function of polar fibrous composites, outside the standard equilibrium conventions, represents an energy contribution that is due to the action of the spherical part of the couple-stress on the relevant fibre curvature-strain field.

Due to the simplicity of the investigated problem though, the obtained solution is based on an equally simple, essentially quadratic displacement field, namely (3.6). All curvature-strain components, which are second-order partial derivatives of the displacement components, are therefore constant, while several of them are equal to zero. It thus happens that zero are also the curvature-strains that combine with the spherical part of the couple-stress in the noted extra energy term that appears in the general form of the curvature part of the strain energy function. This result makes the aforementioned extra energy term zero and thus shows that, due to simplicity, the present example application is essentially an exceptional relevant problem.

Nevertheless, the equilibrium-independent mechanism developed in [12] for the determination of the spherical part of the couple-stress tensor is still applicable in the present case and provides the relevant information sought in a complete and comprehensive manner. Still though, the successful use of this mechanism depends on the appropriateness and effectiveness of the version of polar linear elasticity for fibrous composites that it is applied upon.

It is accordingly seen that the splay-mode/version [17] of the theory (Sect. 4.2) is unable to offer any kind of reliable information, not only regarding the spherical, but also the deviatoric part of the couple-stress tensor. This is because the specific boundary value problem considered is a classical pure bending problem which, as such, does not involve fibre-splay deformation features of any kind. For the same reason, and by essentially recognising that neither fibre-twist characteristics are present, the unrestricted theory (Sect. 4.3) provides information that presents remarkable similarity to that obtained by use of its restricted, bending mode counterpart (Sect. 4.1).

In this context, both the unrestricted theory and its restricted bending mode version predict that only a single component of the deviatoric couple-stress tensor is nonzero, namely $\bar{m}_{13}$. Moreover, both versions of the theory predict that this nonzero $\bar{m}_{13}$-value is constant throughout the plate body. By virtue of a relevant theorem proved in [16], it thus follows that the obtained solution of this polar elasticity problem is unique, in the sense that there exist no additional weak discontinuity solutions (see also [12]). These results further led to the conclusion that the implied bent plate deformation is sustainable in the polar elasticity case only if, in addition to the classical traction boundary conditions assumed in the non-polar elasticity problem (Fig. 2), a constant boundary couple-traction of magnitude $\left|\bar{m}_{13}\right|$ and direction parallel to the $x_{3}$-axis is further applied, in a point-by-point sense, on the opposite edges of the plate that the fibres end upon (Fig. 3).

Moreover, the spherical part of the couple-stress predicted by the unrestricted theory has similar, if not identical qualitative characteristics to those predicted by its restricted, bending mode version. A few, essentially quantitative relevant differences are due to, and arise from the fact that the elastic moduli (4.31) employed in the couple-stress constitutive equations of the unrestricted theory contain advanced material information which enables that model to account not only for fibre-bending, but also for fibre-splay and fibre-twist features of deformation. Nevertheless, this difference between the two versions of the theory does not create substantial mathematical difference in the solution of the present example application, which is exclusively dominated by the bending deformation mode of the plate.

It is fitting in this regard to also note that, in more advanced polar elasticity problems of plate flexure (e.g. [19]), a similarly advanced form of the plate displacement components will return nonzero curvature-strains and will enable them to combine with the spherical part of the couple-stress in a manner that makes also nonzero the aforementioned extra energy term appearing in the curvature part of the strain energy function. As a result, the PDE that corresponds to either (4.12) or (4.42) will be an inhomogeneous one, in the sense that its right-hand side will acquire some specific nonzero form. The general solution of this PDE might thus need to be sought as the superposition of a complementary solution, analogous to either (4.13) or (4.42), and some appropriately specified particular integral/solution of the same.

As the form of such a particular solution is necessarily dictated by the form of the PDE's nonzero right-hand side, the thus obtained general solution will be influenced not only by arbitrary functions of integration, but 
also by specific fibre curvature characteristics that relate to the advanced plate flexure problem considered. Along with an externally imposed set of normal, boundary couple-tractions, analogous to those detailed in (4.36), those fibre curvature characteristics will then also influence the determination process of the involved arbitrary functions of integration.

Open Access This article is licensed under a Creative Commons Attribution 4.0 International License, which permits use, sharing, adaptation, distribution and reproduction in any medium or format, as long as you give appropriate credit to the original author(s) and the source, provide a link to the Creative Commons licence, and indicate if changes were made. The images or other third party material in this article are included in the article's Creative Commons licence, unless indicated otherwise in a credit line to the material. If material is not included in the article's Creative Commons licence and your intended use is not permitted by statutory regulation or exceeds the permitted use, you will need to obtain permission directly from the copyright holder. To view a copy of this licence, visit http://creativecommons.org/licenses/by/4.0/.

\section{Appendix: Solution of the partial differential Eqs. (4.12) and (4.41)}

Potential solution of the PDE (4.12) on the $x_{1} x_{3}$-plane with use of the method of characteristic lines requires initially a search for the plane curves whose tangent satisfies the equation

$$
\frac{\mathrm{d} x_{3}}{\mathrm{~d} x_{1}}=-\frac{S_{11} x_{1}}{4 S_{12} x_{3}} .
$$

Integration of this equation then shows that the characteristic lines sought are the elliptical level curves

$$
\frac{x_{1}^{2}}{4 S_{12}}+\frac{x_{3}^{2}}{S_{11}}=u
$$

If $m_{r r}$ was a function of $x_{1}$ and $x_{3}$ only, the general solution of (4.12) would simply require from $m_{r r}$ to be an arbitrary function of the parameter $u$. However, as $m_{r r}$ may also be a function of $x_{2}$, the solution sought for (4.12) has necessarily the form (4.13) in the three-dimensional space.

If, on the other hand, a potential solution of (4.12) is sought on the $x_{1} x_{3}$-plane with use of the method of the separation of variables, one initially assumes that $m_{r r}$ is of the form

$$
m_{r r}\left(x_{1}, x_{2}, x_{3}\right)=f_{1}\left(x_{1}\right) f_{2}\left(x_{2}\right) f_{3}\left(x_{3}\right) \neq 0 .
$$

Introduction of (4.43) into (4.12), followed by some appropriate rearrangement, leads to

$$
\frac{4 S_{12} f_{1}^{\prime}\left(x_{1}\right)}{x_{1} f_{1}\left(x_{1}\right)}=\frac{S_{11} f_{3}^{\prime}\left(x_{3}\right)}{x_{3} f_{3}\left(x_{3}\right)}=2 g_{2}\left(x_{2}\right)
$$

where a prime denotes ordinary differentiation and, in the $x_{1} x_{3}$-plane, the arbitrary function $2 g_{2}\left(x_{2}\right)$ plays the role of an arbitrary separation variable. Solution of the resulting pair of ordinary differential equations, namely

$$
\frac{\mathrm{d} f_{1}}{f_{1}}=g_{2} \frac{x_{1} \mathrm{~d} x_{1}}{2 S_{12}}, \frac{\mathrm{d} f_{3}}{f_{3}}=2 g_{2} \frac{x_{3} \mathrm{~d} x_{3}}{S_{11}},
$$

yields then (4.14), where the associated arbitrary constants of integration have been incorporated into the arbitrary form of $f_{2}\left(x_{2}\right)$.

Derivation of the general solution (4.42), obtained for the partial differential Eq. (4.41) with the method of characteristics, begins by replacing (4.43) with

$$
\frac{\mathrm{d} x_{3}}{\mathrm{~d} x_{1}}=-\frac{\left(1-2 D_{77} / \hat{d}_{32}\right) S_{11} x_{1}}{S_{12} x_{3}},
$$

and following afterwards identical steps. More specific forms of the general solution (4.42) may also be obtained with use of the method of separation of variables. Starting with (4.45) the search for such solutions will lead to a rather evident generalisation of (4.14) and/or (4.42). 


\section{References}

1. Truesdell, C., Toupin, R.A.: The Classical Field Theories. Encycl. Phys. III/1, 226-793. Springer, Berlin (1960)

2. Mindlin, R.D., Tiersten, H.F.: Effects of couple-stresses in linear elasticity. Arch. Ration. Mech. Anal. 11, 415-448 (1962)

3. Koiter, W.T.: Couple stresses in the theory of elasticity, I and II. Proc. Ned. Akad. Wet. Ser B. 67, 17-44 (1964)

4. Mindlin, R.D., Eshel, E.E.: On first strain-gradient theories in linear elasticity. Int. J. Solids Struct. 4, 109-124 (1968)

5. Eringen, A.C.: Theory of micropolar elasticity. In: Liebowitz, H. (ed.) Fracture, vol. 2, pp. 621-729. Academic Press, New York (1968)

6. Yang, F., Chong, A.C.M., Lam, D.C.C., Tong, P.: Couple stress based strain gradient theory for elasticity. Int. J. Solids Struct. 39, 2731-2743 (2002)

7. Spencer, A.J.M., Soldatos, K.P.: Finite deformations of fibre-reinforced elastic solids with fibre bending stiffness. Int. J. Non-Linear. Mech. 42, 355-368 (2007)

8. Hadjesfandiari, A.R., Dargush, G.F.: Couple stress theory for solids. Int. J. Solids Struct. 48, 24962510 (2011)

9. Soldatos, K.P.: Foundation of polar linear elasticity for fibre-reinforced materials. J. Elast. 114, 155-178 (2014)

10. Neff, P., Münch, I., Ghiba, I.-D., Madeo, A.: On some fundamental misunderstandings in the indeterminate couple stress model. A comment on recent papers of A.R. Hadjesfandiari and G.F. Dargush. Int. J. Solids Struct. 81, 233-243 (2016)

11. Münch, I., Neff, P., Madeo, A., Ghiba, I.-D.: The modified indeterminate couple stress model: Why Yang et al.'s arguments motivating a symmetric couple stress tensor contain a gap and why the couple stress tensor may be chosen symmetric nevertheless. ZAMM 97, 1524-1554 (2017)

12. Soldatos, K.P.: On the characterisation of fibrous composites when fibres resist bending-part III: the spherical part of the couple-stress. Int. J. Solids Struct. 202, 217-225 (2020)

13. Cosserat, E., Cosserat, F.: Théorie des Corps Deformables. Hermann, Paris (1909)

14. Timoshenko, S.P., Goodier, J.N.: Theory of Elasticity, 3rd edn. McGraw-Hill, New York (1970)

15. Love, A.E.H.: A Treatise on the Mathematical Theory of Elasticity, Reprint Dover Publ, New York (1944)

16. Soldatos, K.P.: On the characterisation of fibrous composites when fibres resist bending. Int. J. Solids Struct. 146-149, 35-43 (2018)

17. Soldatos, K.P., Shariff, M.H.B.M., Merodio, J.: On the constitution of polar fibre-reinforced materials. Mech. Adv. Mater. Struct., ISSN: 1537-6494 1537-6532 (Available online). https://doi.org/10.1080/15376494.2020.1729449 (2020)

18. Jones, R.M.: Mechanics of Composite Materials. Taylor \& Francis, Washington (1996)

19. Farhat, A.F.: Basic Problems of Fibre-reinforced Structural Components when Fibres Resist Bending. PhD Thesis, Sch. Math. Sci., University of Nottingham, Nottingham (2013).

Publisher's Note Springer Nature remains neutral with regard to jurisdictional claims in published maps and institutional affiliations. 